\title{
Superconvergence of a Finite Element Approximation to the Solution of a Sobolev Equation in a Single Space Variable
}

\author{
By Douglas N. Arnold, Jim Douglas, Jr. and Vidar Thomée*
}

\begin{abstract}
A standard Galerkin method for a quasilinear equation of Sobolev type using continuous, piecewise-polynomial spaces is presented and analyzed. Optimal order error estimates are established in various norms, and nodal superconvergence is demonstrated. Discretization in time by explicit single-step methods is discussed.
\end{abstract}

1. Introduction. We shall consider the numerical solution of the periodic initial value problem for the Sobolev equation given by

$$
\begin{gathered}
-\left(a u_{x t}\right)_{x}+c u_{t}=-\left(\alpha u_{x}\right)_{x}+\beta u_{x}+\gamma \quad \text { for } x \in \mathbf{R}, t \in J=[0, T], \\
u(x, 0)=u_{0}(x) \quad \text { for } x \in \mathbf{R}, \\
u(x+1, t)=u(x, t) \quad \text { for }(x, t) \in \mathbf{R} \times J .
\end{gathered}
$$

The coefficients $a$ and $c$ will be allowed to depend on $x$ and will be assumed to be 1 -periodic and bounded above and below by positive constants, so that the bilinear form

$$
A(\varphi, \psi)=\left(a \varphi^{\prime}, \psi^{\prime}\right)+(c \varphi, \psi)=\int_{0}^{1}\left(a \varphi^{\prime} \psi^{\prime}+c \varphi \psi\right) d x
$$

is equivalent to the usual inner product on the Sobolev space $H^{1}([0,1])$. The coefficients $\alpha, \beta$, and $\gamma$ will be $C^{1}$ functions of $x, t$ and $u$ which are 1-periodic with respect to $x$.

Sobolev equations of the form (1.1a) have been employed to model a variety of physical processes (see [6] for references). An example of particular interest is the equation

$$
-u_{x x t}+u_{t}+(1+u) u_{x}-\nu u_{x x}=0
$$

that has been studied extensively by Benjamin, Bona, and Mahony [1] and others [3], [13]-[16] as an alternative to the Korteweg-de Vries equation for describing unidirectional, long, dispersive waves.

The numerical solution of problems similar to (1.1) has been treated by a number of authors [5]-[9], using both finite difference and finite element methods. The special case described by (1.2) has also been studied in [2], [4], [16], [17], [20]. We shall consider a standard Galerkin method for (1.1), and we shall derive both

Received December 21, 1979.

1980 Mathematics Subject Classification. Primary 65N30.

* The research of Professor Douglas was supported in part by the National Science Foundation and the Laboratorio de Cálculo/CBPF, Rio de Janeiro and that of Professor Thomee in part by the National Science Foundation. 0025-5718/81/0000-0004/\$03.75 
global estimates and, more interestingly, superconvergence results for the approximate solution at knots at which the smoothness constraint of the piecewisepolynomial trial space reduces to simple continuity. Our global estimates, while new in their explicit form, are closely related to ones obtained earlier by Ewing [6]; they are included so that they can be applied in the derivation of the superconvergence estimates. The superconvergence is demonstrated using a duality argument based on the pivot space formed by the periodic functions in $H^{1}$.

Denote the periodic Sobolev spaces by

$$
H_{p}^{k}=\left\{f \in H_{\mathrm{loc}}^{k}(\mathbf{R}) \mid f(x+1)=f(x), x \in \mathbf{R}\right\},
$$

and let the norm of $H_{p}^{k}$ be $\|f\|_{k}=\|f\|_{H^{k}(I)}$, where $I$ is any interval of length one. Frequently, the index zero will be omitted for $H^{0}(I)=L^{2}(I)$. We shall abbreviate the notation for the space $L^{q}\left(0, T ; H_{p}^{k}\right)$ to $L^{q}\left(H_{p}^{k}\right), 1 \leqslant q \leqslant \infty$. The letter $C$ will be used to indicate generic constants, and the usual functional notation will be employed to specify dependence. However, dependence on $T$ and the coefficients $a, c, \alpha, \beta$, and $\gamma$ will generally not be noted explicitly.

We assume that the problem (1.1) is well-posed in the sense that, for given $u_{0} \in H_{p}^{1}$, there exists a unique $C^{1}$ map $u$ of $J$ into $H_{p}^{1}$ satisfying (1.1a) weakly and such that $u(0)=u_{0}$. Existence and uniqueness results of this sort can be proved under a variety of hypotheses. Well-posedness of (1.2) is proved in [1], [3], [13], [14], [15], [18]. The following theorem, the proof of which is indicated in the Appendix, is similar, but not identical, to several appearing elsewhere [10], [18], [19].

THEOREM 1.1. Suppose that $a$ and $c$ are strictly positive, 1-periodic functions in $L^{\infty}(\mathbf{R})$ and that $\alpha, \beta, \gamma \in C_{b}^{1}(\mathbf{R} \times J \times \mathbf{R})$ are 1-periodic with respect to their first argument. Then, given $u_{0} \in H_{p}^{1}$, there exists a unique $C^{1}$ map $u$ of $J$ into $H_{p}^{1}$ such that

$$
\begin{gathered}
A\left(u_{t}, \chi\right)=\left(\alpha(u) u_{x}, \chi_{x}\right)+\left(\beta(u) u_{x}, \chi\right)+(\gamma(u), \chi) \text { for } \chi \in H_{p}^{1}, \\
u(0)=u_{0} .
\end{gathered}
$$

Further, $\|u\|_{L^{\infty}\left(H_{\rho}^{\prime}\right)}$ is bounded by a constant depending only on $\left\|u_{0}\right\|_{1}, T$, and the coefficients $a, c, \alpha, \beta$, and $\gamma$.

The following regularity theorem is also proved in the Appendix.

THEOREM 1.2. Let $k$ be a positive integer, let $m=\max (1, k-1)$, and suppose that $a$ and $c$ are strictly positive, 1-periodic functions in $C^{k-1}(\mathbf{R})$, and that $\alpha, \beta$, and $\gamma$ are functions in $C^{m}(\mathbf{R})$ which are 1-periodic with respect to their first argument. Let $u$ be $a C^{1}$ map of $J$ into $H_{p}^{1}$ satisfying (1.3) with $u_{0} \in H_{p}^{k}$. Then $u(t) \in H_{p}^{k}$ for all $t \in J$. Moreover

$$
\|u\|_{L^{\infty}\left(H_{p}^{k}\right)}+\left\|u_{t}\right\|_{L^{\infty}\left(H_{p}^{k}\right)}<C,
$$

where $C$ is a constant which depends only on $\left\|u_{0}\right\|_{k}, T$, and the coefficients $a, c, \alpha, \beta$, and $\gamma$.

2. The Finite Element Approximation. We suppose given a sequence of values of $h$ clustering at 0 and for each $h$ a partition $0=x_{0}^{h}<x_{1}^{h}<\cdots<x_{n_{h}}^{h}=1$ with $\sup _{i}\left(x_{i}^{h}-x_{i-1}^{h}\right)=h$. The finite element space $\mathfrak{N}=\mathfrak{N}_{h} \subset H_{p}^{1}$ is then taken to be the space of continuous, 1-periodic functions which restrict to polynomials of 
degree at most $r$ on each of the subintervals $\left(x_{i-1}^{h}, x_{i}^{h}\right)$. Here, $r>1$ is a fixed integer. It is well known that for some constant $C$, independent of $h$,

$$
\inf _{\chi \in \mathscr{T}_{h}}\left(\|\varphi-\chi\|+h\|\varphi-\chi\|_{1}\right) \leqslant C\|\varphi\|_{q} h^{q} \quad \text { for } \varphi \in H_{p}^{q}, 1 \leqslant q \leqslant r+1 .
$$

The semidiscrete solution of (1.1) is defined to be the function $U=U_{h}: J \rightarrow \mathfrak{N}$ such that

$$
\begin{gathered}
A\left(U_{t}, \chi\right)=\left(\alpha(U) U_{x}, \chi_{x}\right)+\left(\beta(U) U_{x}, \chi\right)+(\gamma(U), \chi) \text { for } \chi \in \Re, t \in J, \\
A(U(0), \chi)=A\left(u_{0}, \chi\right) \text { for } \chi \in \Re
\end{gathered}
$$

The Eqs. (2.2a) can be interpreted as a finite system of ordinary differential equations in the coefficients of $U$ with respect to some basis for $\mathfrak{T}$. Therefore, (2.2) has a unique solution at least locally in $t$. In the next section we shall show that the solution $U$ persists for $t \in J$.

3. Global Error Estimates. Henceforth, we assume that $a, c \in C^{r}(\mathbf{R})$ and $\alpha, \beta, \gamma$ $\in C^{r}(\mathbf{R} \times J \times \mathbf{R})$. In this section we prove the following theorem.

THEOREM 3.1. The solution $U_{h}$ of (2.2) is defined for all $t \in J$, and

$$
\left\|U_{h}-u\right\|_{L^{\infty}\left(H_{p}^{s}\right)} \leqslant C\left(\left\|u_{0}\right\|_{q}\right) h^{q-s} \text { for } 0 \leqslant s \leqslant 1<q \leqslant r+1 .
$$

Proof. First we make the temporary assumption that $\alpha, \beta, \gamma \in C_{b}^{1}(\mathbf{R} \times J \times \mathbf{R})$. It is then easy to see that $U$ is defined on all of $J$. Indeed, setting $\chi=U$ in (2.2a) and integrating over the interval $(0, t) \subset J$ yields

$$
A(U(t), U(t)) \leqslant A(U(0), U(0))+C \int_{0}^{t}\|U(\tau)\|_{1}^{2} d \tau .
$$

From this relation, Gronwall's lemma and (2.2b) follow the a priori estimates

$$
\|U\|_{L^{\infty}\left(H_{p}^{\prime}\right)} \leqslant C\|U(0)\|_{1} \leqslant C\left\|u_{0}\right\|_{1}
$$

which suffice to establish existence of $U(t)$ for all $t \in J$.

For the purpose of the error analysis, we introduce the elliptic projection $\tilde{u}=\tilde{u}_{h}$ : $J \rightarrow \mathfrak{N}$ of $u$, defined by the equations

$$
A(\tilde{u}, \chi)=A(u, \chi) \text { for } \chi \in \Re \text {. }
$$

Let $\eta=\tilde{u}-u, \zeta=U-u, \xi=\tilde{u}-U=\eta-\zeta$. It is well known that

$$
\|\eta(t)\|_{s} \leqslant C\|u(t)\|_{q} h^{q-s} \text { for } t \in J, 0<s<1<q<r+1 .
$$

Hence, in order to prove the theorem, it suffices to show that

$$
\|\xi\|_{L^{\infty}\left(H_{p}^{s}\right)} \leqslant C\left(\left\|u_{0}\right\|_{q}\right) h^{q-s} \text { for } 0 \leqslant s \leqslant 1 \leqslant q \leqslant r+1 .
$$

Now, if we subtract the weak form of (1.1a) from (2.2a) and use Taylor's theorem, we find that

$$
\begin{aligned}
A\left(\zeta_{t}, \chi\right)= & B(\zeta, \chi) \equiv\left(\alpha(U) \zeta_{x}, \chi_{x}\right)+\left(\bar{\alpha}_{u} u_{x} \zeta, \chi_{x}\right) \\
& +\left(\beta(U) \zeta_{x}, \chi\right)+\left(\left[\bar{\beta}_{u} u_{x}+\bar{\gamma}_{u}\right] \zeta, \chi\right) \text { for } \chi \in \mathfrak{N},
\end{aligned}
$$

where $\bar{F}_{u}=\int_{0}^{1} F_{u}(u+\tau \zeta) d \tau$ for $F=\alpha, \beta$, or $\gamma$. Note that, since the solution $u$ of (1.1) is stable in the space $H_{p}{ }^{1}$ by Theorem 1.1 and since $\|\varphi\|_{L^{\infty}}<\|\varphi\|_{1}$,

$$
|B(\varphi, \psi)| \leqslant C\left(\left\|u_{0}\right\|_{1}\right)\|\varphi\|_{1}\|\psi\|_{1} \quad \text { for } \varphi, \psi \in H_{p}^{1} \text {. }
$$


Moreover, integrating by parts, we obtain

$$
\begin{array}{r}
B(\varphi, \psi)=\left(\varphi,-\alpha(U) \psi_{x x}+\left[-\alpha_{x}(U)-\alpha_{u}(U) U_{x}+\bar{\alpha}_{x} u_{x}-\beta(U)\right] \psi_{x}\right. \\
\left.+\left[-\beta_{x}(U)-\beta_{u}(U) U_{x}+\bar{\beta}_{u} u_{x}+\bar{\gamma}_{u}\right] \psi\right)
\end{array}
$$

Since (3.1) implies that

$\left|\left(\varphi, \alpha_{u}(U) U_{x} \psi_{x}\right)\right| \leqslant\|\varphi\|\left\|\alpha_{u}(U)\right\|_{L^{\infty}}\left\|U_{x}\right\|\left\|\psi_{x}\right\|_{L^{\infty}} \leqslant C\left(\left\|u_{0}\right\|_{1}\right)\|\varphi\|\|\psi\|_{2}$, then

$$
|B(\varphi, \psi)| \leqslant C\left(\left\|u_{0}\right\|_{1}\right)\|\varphi\|\|\psi\|_{2}
$$

It follows from (3.2) that $A\left(\eta_{t}, \chi\right)=0$ for $\chi \in \Re$; consequently, $A\left(\xi_{t}, \chi\right)=$ $-A\left(\zeta_{t}, \chi\right)$ and

$$
A\left(\xi_{t}, \chi\right)=-B(\zeta, \chi) \text { for } \chi \in \mathfrak{T}
$$

The initial values of $U_{h}$ were chosen in (2.2b) so that

$$
\xi(0)=0 \text {. }
$$

The choice $\chi=\xi_{t}$ in (3.7a) leads to the inequality

$$
\left\|\xi_{t}\right\|_{1} \leqslant C\left(\left\|u_{0}\right\|_{1}\right)\|\zeta\|_{1}
$$

Now, by (3.7b),

$$
\begin{aligned}
\|\xi(t)\|_{1} & =\left\|\int_{0}^{t} \xi_{t}(\tau) d \tau\right\|_{1} \leqslant \int_{0}^{t}\left\|\xi_{t}(\tau)\right\|_{1} d \tau \\
& \leqslant C\left(\left\|u_{0}\right\|_{1}\right) \int_{0}^{t}\left(\|\xi(\tau)\|_{1}+\|\eta(\tau)\|_{1}\right) d \tau
\end{aligned}
$$

since $\zeta=\eta-\xi$. An application of Gronwall's lemma shows that

$$
\|\xi\|_{L^{\infty}\left(H_{p}^{\prime}\right)} \leqslant C\left(\left\|u_{0}\right\|_{1}\right)\|\eta\|_{L^{\prime}\left(H_{p}^{\prime}\right)} \leqslant C\left(\left\|u_{0}\right\|_{1}\right)\|u\|_{L^{\prime}\left(H_{p}^{q}\right)} h^{q-1} .
$$

In light of the regularity Theorem 1.2, this shows the desired estimate for $s=1$.

It remains to consider the case $s=0$. Fix $t \in J$ and let $\psi \in H_{p}^{2}$ be the 1-periodic solution of the equation

$$
-\left(a \psi_{x}\right)_{x}+c \psi=\xi_{t}(t)
$$

Then,

$$
\|\psi\|_{2} \leqslant C\left\|\xi_{t}(t)\right\|
$$

Now, by (3.7a),

$$
\begin{aligned}
\left\|\xi_{t}(t)\right\|^{2} & =A\left(\xi_{t}(t), \psi\right) \\
& =A\left(\xi_{t}(t), \psi-\chi\right)+B(\zeta(t), \psi-\chi)-B(\zeta(t), \psi) \text { for } \chi \in \Re
\end{aligned}
$$

So, by (3.5) and (3.6),

$$
\left\|\xi_{t}(t)\right\|^{2} \leqslant C\left[\left(\left\|\xi_{t}(t)\right\|_{1}+\|\zeta(t)\|_{1}\right) \inf _{\chi \in \mathscr{R}}\|\psi-\chi\|_{1}+\|\zeta(t)\|\|\psi\|_{2}\right],
$$

with $C=C\left(\left\|u_{0}\right\|_{1}\right)$. Hence, by (2.1), (3.8), and (3.9),

$$
\left\|\xi_{t}(t)\right\| \leqslant C\left(\|\zeta(t)\|_{1} h+\|\zeta(t)\|\right) \leqslant C\left(\left\|u_{0}\right\|_{q}\right)\left(h^{q}+\|\xi(t)\|\right),
$$

where in the latter inequality we have used the $H^{1}$ estimate on $\xi$ and $H^{1}$ and $L^{2}$ estimates on $\eta$. Integration in time and application of Gronwall's lemma as before yields the desired estimate and thus completes the proof of the theorem in case $\alpha, \beta, \gamma \in C_{b}^{1}(\mathbf{R} \times J \times \mathbf{R})$. 
Finally, we must remove the hypothesis that the coefficients and their first derivatives are bounded. Let $\alpha^{*}, \beta^{*}$, and $\gamma^{*}$ be functions in $C_{b}^{1}(\mathbf{R} \times J \times \mathbf{R})$ which are periodic with respect to their first argument, and which extend $\alpha, \beta$, and $\gamma$ from a neighborhood of the solution surface, $\{(x, t, u(x, t)) \mid(x, t) \in \mathbf{R} \times J\}$. Then the differential problem derived from (1.1) by replacing the coefficients $\alpha, \beta, \gamma$ with $\alpha^{*}$, $\beta^{*}, \gamma^{*}$ has a unique solution by Theorem 1.1 , which must be $u$. The above analysis applies to that equation, giving for each $h$ the semidiscrete Galerkin solution $U_{h}^{*}$, which converges to $u$ uniformly in $(x, t)$. For small $h,\left(\alpha^{*}, \beta^{*}, \gamma^{*}\right)=(\alpha, \beta, \gamma)$ on $\left\{\left(x, t, U_{h}^{*}(x, t)\right) \mid(x, t) \in \mathbf{R} \times J\right\}$, and so $U_{h}^{*}$ must also be the (necessarily unique) semidiscrete Galerkin solution for the original problem; i.e., $U_{h}=U_{h}^{*}$ converges to $u$ and the desired error estimates hold.

4. Superconvergence Estimates. The following theorem is the major result of this paper. It will be deduced by a refinement of the duality argument used in showing the $L^{2}$-estimate in Theorem 3.1.

THEOREM 4.1. The error in the solution given by the semidiscrete finite element method satisfies the inequality

$$
\left|U_{h}(\bar{x}, t)-u(\bar{x}, t)\right| \leqslant C\left(\left\|u_{0}\right\|_{r+1}\right) h^{2 r}
$$

for each knot $\bar{x} \in\left\{x_{0}^{h}, x_{1}^{h}, \ldots, x_{n_{h}}^{h}\right\}$ and each $t \in J$.

For the proof, we fix a knot $\bar{x}$ and define the space

$$
\tilde{H}=H_{p}^{1} \cap H^{r+1}((\bar{x}, \bar{x}+1)),
$$

normed by $\|\psi \psi\|=\|\psi\|_{H^{r+1}((\bar{x}, \bar{x}+1))}$. Thus, the elements of $\tilde{H}$ are continuous 1-periodic functions with $r+1$ locally square-integrable derivatives, except at the point $\bar{x}$, where the first derivative can have a simple discontinuity. An example of such a function is the periodic Green's function for the operator $\varphi \rightarrow-\left(a \varphi_{x}\right)_{x}+c \varphi$ at $\bar{x}$; that is, the function $G \in H_{p}{ }^{1}$ defined by the equations

$$
A(\varphi, G)=\varphi(\bar{x}) \text { for } \varphi \in H_{p}^{1}
$$

From the elementary theory of ordinary differential equations we know that $\|G\| \|$ may be bounded by a constant depending only on $a$ and $c$.

Since the finite element space is constrained only by continuity at the knot $\bar{x}$, the usual proof of (2.1) yields the estimate

$$
\inf _{\chi \in \mathscr{R}}\|\psi-\chi\|_{1} \leqslant C\|\psi\| h^{r} \quad \text { for } \psi \in \tilde{H} .
$$

For $\varphi \in H_{p}^{1}$, we define the following dual norm:

$$
\|\varphi\|_{*}=\sup _{0 \neq \psi \in \tilde{H}} A(\varphi, \psi) /\|\psi\| \| .
$$

The lemma below shows that this norm dominates the knot value.

LEMMA 4.2. There exists a constant $C$ such that

$$
|\varphi(\bar{x})| \leqslant C\|\varphi\|_{*} \text { for } \varphi \in H_{p}^{1} .
$$

Proof. This follows immediately from the relations

$$
|\varphi(\bar{x})|=|A(\varphi, G)| \leqslant\left\|\varphi \left|\left\|_{*} \mid\right\| G\|\| .\right.\right.
$$


Now ||$\cdot|| \mid$ has the appearance of a norm of order $r+1$, and we have defined its dual by pivoting about the $H_{p}^{1}$ inner product; so, we should expect $\||\cdot|\|_{*}$ to behave like a norm of order $-(r-1)$. (In fact, as we shall see at the end of this section, $\|\mid \cdot\|_{*}$ dominates the usual norm in $H_{p}^{-(r-1)}$.) Thus, the exponent appearing in the next result is the expected one.

LEMMA 4.3. The dual norm of the error $\eta=\tilde{u}_{h}-u$ in the elliptic projection satisfies the inequality

$$
\|\eta(t)\|_{*} \leqslant C\left(\left\|u_{0}\right\|_{r+1}\right) h^{2 r} \text { for } t \in J .
$$

Proof. Let $\psi \in \tilde{H}$. By (3.3) and (4.1),

$$
A(\eta, \psi)=\inf _{\chi \in \mathscr{R}} A(\eta, \psi-\chi) \leqslant C\|\eta\|_{1} \inf _{\chi \in \mathscr{R}}\|\psi-\chi\|_{1}<C\|u\|_{r+1} h^{2 r}\|\psi\| \|,
$$

which demonstrates the lemma.

To bound $\|\xi\|_{*}$ we need a lemma which will allow us to estimate the right-hand side of (3.7a) by duality. Since the coefficients of the form $B$ are not known to be sufficiently smooth to enable us to do this, we first refine this form. Carrying some additional terms in the Taylor expansions, we write (3.7a) in the form

$$
A(\xi, \chi)=-\bar{B}(\zeta, \chi)-R(\chi) \text { for } \chi \in \mathfrak{N},
$$

where

$$
\begin{aligned}
\bar{B}(\varphi, \psi)= & \left(\alpha(u) \varphi_{x}, \psi_{x}\right)+\left(\alpha_{u}(u) u_{x} \varphi, \psi_{x}\right) \\
& +\left(\beta(u) \varphi_{x}, \psi\right)+\left(\left[\beta_{u}(u) u_{x}+\gamma_{u}(u)\right] \varphi, \psi\right)
\end{aligned}
$$

and

$$
R(\chi)=\left(\bar{\alpha}_{u} \zeta \zeta_{x}+\bar{\alpha}_{u u} u_{x} \zeta^{2}, \chi_{x}\right)+\left(\bar{\beta}_{u} \zeta \zeta_{x}+\bar{\beta}_{u u} u_{x} \zeta^{2}+\bar{\gamma}_{u u} \zeta^{2}, \chi\right)
$$

Here,

$$
\bar{F}_{u u}=\int_{0}^{1}(1-\tau) F_{u u}(u+\tau(U-u)) d \tau \quad \text { for } F=\alpha, \beta \text {, or } \gamma .
$$

Note that, by Theorem 3.1,

$$
|R(\chi)| \leqslant C\|\zeta\|_{L^{\infty}}\|\zeta\|_{1}\|\chi\|_{1} \leqslant C\left(\left\|u_{0}\right\|_{r+1}\right) h^{2 r}\|x\|_{1} .
$$

Clearly,

$$
|\bar{B}(\varphi, \psi)| \leqslant C\left(\left\|u_{0}\right\|_{1}\right)\|\varphi\|_{1}\|\psi\|_{1} .
$$

LEMMA 4.4. There exists $C=C\left(\left\|u_{0}\right\|_{r+1}\right)$ such that

$$
|\bar{B}(\varphi, \psi)|<C\|\varphi\|_{*}\|\psi \mid\| \text { for } \varphi \in H_{p}^{1}, \psi \in \tilde{H}, t \in J .
$$

Proof. It follows from (4.4) and the Riesz representation theorem that, for given $\psi \in H_{p}^{1}$, there exists a unique $\bar{\psi} \in H_{p}{ }^{1}$ such that

$$
A(\varphi, \bar{\psi})=\bar{B}(\varphi, \psi) \text { for } \varphi \in H_{p}^{1}
$$

and

$$
\|\bar{\psi}\|_{1} \leqslant C\left(\left\|u_{0}\right\|_{1}\right)\|\psi\|_{1}
$$

Clearly, it now suffices to show that

$$
\|\bar{\psi}|\|<C\| \psi|\| \text { for } \psi \in \tilde{H} .
$$


In view of the definition of $\bar{B}, \bar{\psi}$ satisfies the differential equation

$-\left(a \bar{\psi}_{x}\right)_{x}+c \bar{\psi}=\theta \equiv-\left(\alpha(u) \psi_{x}\right)_{x}+\alpha_{u}(u) u_{x} \psi_{x}-(\beta(u) \psi)_{x}+\left(\beta_{u}(u) u_{x}+\gamma_{u}(u)\right) \psi$ on $(\bar{x}, \bar{x}+1)$. Moreover,

$$
|\bar{\psi}(\bar{x})|=|\bar{\psi}(\bar{x}+1)| \leqslant\|\bar{\psi}\|_{1} \leqslant C\left(\left\|u_{0}\right\|_{1}\right)\|\psi\|_{1} .
$$

Thus, regularity of the Dirichlet two-point boundary value problem implies that

$$
\|\bar{\psi}\| \leqslant C\left(\left\|u_{0}\right\|_{1}\right)\left(\|\psi\|_{1}+\|\theta\|_{H^{r-1}((\bar{x}, \bar{x}+1))}\right) \leqslant C\left(\left\|u_{0}\right\|_{r+1}\right)\|\psi\| \|,
$$

which shows (4.5).

Proof of Theorem 4.1. By Lemmas 4.2 and 4.3 it remains to show that, for $\xi=\tilde{u}-U_{h}$,

$$
\|\xi\|_{*} \leqslant C\left(\left\|u_{0}\right\|_{r+1}\right) h^{2 r} \quad \text { for } t \in J .
$$

Let $\psi \in \tilde{H}$. By (4.2), we have for fixed $t \in J$ the relation

$$
A\left(\xi_{t}, \psi\right)=A\left(\xi_{t}, \psi-\chi\right)+\bar{B}(\zeta, \psi-\chi)-\bar{B}(\zeta, \psi)-R(\chi)
$$

Choosing $\chi$ by (4.1) and using (4.4), Lemma 4.4, and (4.3), we obtain the estimate

$$
\left|A\left(\xi_{t}, \psi\right)\right| \leqslant C\left(\left\|u_{0}\right\|_{r+1}\right)\left(\left(\left\|\xi_{t}\right\|_{1}+\|\zeta\|_{1}\right) h^{r}+\|\mid \zeta\|_{*}+h^{2 r}\right)\|\psi \mid\| .
$$

Thus,

$$
\left\|\xi_{t}\right\|_{*} \leqslant C\left(\left\|u_{0}\right\|_{r+1}\right)\left(h^{2 r}+\|\xi\|_{*}\right),
$$

by Lemma 4.3, (3.8), and Theorem 3.1. Integrate (4.7) in time and apply Gronwall's lemma to conclude (4.6). Hence, the theorem has been proved.

We shall complete this section by demonstrating that, for $\varphi \in H_{p}^{1}$,

$$
\|\varphi\|_{H_{p}^{(r-1)}} \equiv \sup _{\psi \in H_{p}^{r-1}}(\varphi, \psi) /\|\psi\|_{r-1} \leqslant C\|\varphi\|_{*},
$$

and thus, by Lemma 4.3 and (4.6),

$$
\left\|U_{h}-u\right\|_{L^{\infty}\left(H_{\rho}^{-(r-1)}\right)} \leqslant C\left(\left\|u_{0}\right\|_{r+1}\right) h^{2 r} .
$$

In fact, for given $\psi \in H_{p}^{r-1}$, there exists a unique $\bar{\psi} \in H_{p}^{r+1}$ such that

$$
(\varphi, \psi)=A(\varphi, \bar{\psi}) \text { for } \varphi \in H_{p}^{1},
$$

and

$$
\|\bar{\psi}\|=\|\bar{\psi}\|_{r+1} \leqslant C\|\psi\|_{r-1} .
$$

Hence, by the definition of $\||\cdot|\|_{*}$,

$$
|(\varphi, \psi)| \leqslant\|\varphi\|_{*}\|\bar{\psi}\||\leqslant C|\|\varphi\|_{*}\|\psi\|_{r-1},
$$

which shows (4.8).

5. Discretization in Time by Explicit Single-Step Methods. When solving parabolic differential equations by finite element methods, one typically uses an implicit finite difference method to solve the system of ordinary differential equations which arises from the finite element discretization of the spatial operator. Standard explicit ODE solvers do not generally provide stable approximations if the time step and the mesh parameter are allowed to tend to zero independently. 
For Sobolev equations, however, the situation is more favorable. In fact, the usual bounds for the truncation error of single-step methods, such as Runge-Kutta methods or predictor-corrector methods based on quadrature rules, apply equally well to the semidiscrete Sobolev equation (2.2), with the constant independent of the level of spatial discretization. We do not wish to carry out the error analysis for such methods at length here and so shall consider only the simple case of Euler's method. Convergence proofs for more accurate methods, as described in, e.g., [11], can easily be adapted to our situation in the same manner.

The Euler-Galerkin method defines approximations $U^{n}$ to $u$ at $t_{n}=n k(k>0$ being the step-size) by the equations

$$
\begin{aligned}
& A\left(U^{n+1}-U^{n}, \chi\right)=k\left[\left(\alpha\left(t_{n}, U^{n}\right) U_{x}^{n}, \chi_{x}\right)\right. \\
& \left.+\left(\beta\left(t_{n}, U^{n}\right) U_{x}^{n}, \chi\right)+\left(\gamma\left(t_{n}, U^{n}\right), \chi\right)\right] \text { for } \chi \in \mathfrak{N}, \\
& A\left(U^{0}, \chi\right)=A\left(u_{0}, \chi\right) \text { for } \chi \in \mathfrak{R} \text {. }
\end{aligned}
$$

THEOREM 5.1. The error in the Euler-Galerkin method satisfies the following inequalities:

$$
\begin{aligned}
\sup _{n=0,1, \ldots,[T / k]}\left\|U^{n}-u\left(t_{n}\right)\right\|_{s} \leqslant C\left(\left\|u_{0}\right\|_{q}\right) h^{q-s}+C\left(\left\|u_{0}\right\|_{1}\right) k & \\
& \text { for } 0 \leqslant s \leqslant 1 \leqslant q \leqslant r+1,
\end{aligned}
$$

and, with $\bar{x} \in\left\{x_{0}^{h}, x_{1}^{h}, \ldots, x_{n_{h}}^{h}\right\}$,

$$
\sup _{n=0,1, \ldots,[T / k]}\left|U^{n}(\bar{x})-u\left(\bar{x}, t_{n}\right)\right| \leqslant C\left(\left\|u_{0}\right\|_{r+1}\right) h^{2 r}+C\left(\left\|u_{0}\right\|_{1}\right) k .
$$

Proof. In view of Theorems 3.1 and 4.1, it suffices to show that, for $e^{n}=U^{n}-$ $U\left(t_{n}\right)$,

$$
\left\|e^{n}\right\|_{1} \leqslant C\left(\left\|u_{0}\right\|_{1}\right) k \text { for } n=1,2, \ldots,[T / k]
$$

Since

$$
U\left(t_{n+1}\right)-U\left(t_{n}\right)=k U_{t}\left(t_{n}\right)+k^{2} \int_{0}^{1} U_{t t}\left(t_{n}+\tau k\right)(1-\tau) d \tau,
$$

then, by (2.2),

$$
\begin{aligned}
& A\left(U\left(t_{n+1}\right)-U\left(t_{n}\right), \chi\right) \\
& =k\left[\left(\alpha\left(t_{n}, U\left(t_{n}\right)\right) U_{x}\left(t_{n}\right), \chi_{x}\right)+\left(\beta\left(t_{n}, U\left(t_{n}\right)\right) U_{x}\left(t_{n}\right), \chi\right)+\left(\gamma\left(t_{n}, U\left(t_{n}\right)\right), \chi\right)\right] \\
& \quad+k^{2} \int_{0}^{1} A\left(U_{t t}\left(t_{n}+\tau k\right), \chi\right)(1-\tau) d \tau \text { for } \chi \in \Re
\end{aligned}
$$

We now subtract (5.2) from (5.1a) and use the bound (invoke again the argument at the end of Section 3 to avoid global boundedness assumptions)

$$
\left\|F\left(t_{n}, U^{n}\right)-F\left(t_{n}, U\left(t_{n}\right)\right)\right\|_{L^{\infty}} \leqslant C\left\|e^{n}\right\|_{L^{\infty}} \leqslant C\left\|e^{n}\right\|_{1} \text { for } F=\alpha, \beta \text {, or } \gamma,
$$

and the fact that (cf. (A.2) and (A.3))

$$
\|U\|_{L^{\infty}\left(H_{p}^{\prime}\right)}+\left\|U_{t t}\right\|_{L^{\infty}\left(H_{p}^{1}\right)} \leqslant C\left(\left\|u_{0}\right\|_{1}\right) .
$$

Then,

$$
\left|A\left(e^{n+1}-e^{n}, \chi\right)\right| \leqslant C\left(\left\|u_{0}\right\|_{1}\right)\left(k\left\|e^{n}\right\|_{1}+k^{2}\right)\|\chi\|_{1} \quad \text { for } \chi \in \mathfrak{N}
$$


It results from the choice $\chi=e^{n+1}$ that

$$
A\left(e^{n+1}, e^{n+1}\right)^{1 / 2} \leqslant(1+C k) A\left(e^{n}, e^{n}\right)^{1 / 2}+C k^{2} .
$$

Since $e^{0}=0$, a simple induction shows that

$$
\begin{aligned}
\left\|e^{n}\right\|_{1} \leqslant C_{1} A\left(e^{n}, e^{n}\right)^{1 / 2} \leqslant C_{1}\left((1+C k)^{n}-1\right) k \leqslant C_{1}\left(e^{C T}-1\right) k, \\
\quad \text { for } n=0,1, \ldots,[T / k],
\end{aligned}
$$

which completes the proof of the theorem.

Note that to solve (5.1) it is necessary only to factor and store the single band matrix corresponding to the form $A$.

\section{Appendix.}

Proof of Theorem 1.1. The method of Faedo-Galerkin [12] can be applied in an essentially standard way. Letting $\left\{\chi^{m}\right\}_{m=1}^{\infty}$ be a smooth basis for $H_{p}^{1}$ and $V^{n}$ the linear span of $\left\{\chi^{m}\right\}_{m=1}^{n}$, we define $t^{n} \in(0, T]$ and $u^{n} \in C^{1}\left(0, t^{n} ; V^{n}\right)$ so that

$$
\begin{aligned}
& A\left(u_{t}^{n}, \chi^{m}\right)=\left(\alpha\left(u^{n}\right) u_{x}^{n}, \chi_{x}^{m}\right)+\left(\beta\left(u^{n}\right) u_{x}^{n}, \chi^{m}\right)+\left(\gamma\left(u^{n}\right), \chi^{m}\right) \\
& \text { for } t \in\left[0, t^{n}\right], m=1,2, \ldots, n \text {, } \\
& A\left(u^{n}(0), \chi^{m}\right)=A\left(u_{0}, \chi^{m}\right) \text { for } m=1,2, \ldots, n,
\end{aligned}
$$

which is possible by the standard existence theorem for ordinary differential equations. Replacing $\chi^{m}$ by $u^{n}$, we find that

$$
\left\|u^{n}(t)\right\|_{1} \leqslant C \text { for } t \in\left[0, t^{n}\right]
$$

with $C$ depending only on $a, c, \alpha, \beta, \gamma, T$, and $\left\|u_{0}\right\|_{1}$ but not on $n$. It follows that we may take $t^{n}=T$ for all $n$. Next, using the test function $u_{t}^{n} \in V^{n}$ we conclude that

$$
\left\|u_{t}^{n}\right\|_{L^{\infty}\left(H_{p}^{1}\right)} \leqslant C\left\|u^{n}\right\|_{L^{\infty}\left(H_{p}^{1}\right)} \leqslant C,
$$

and, by differentiating (A.1) and substituting $u_{t t}^{n} \in V^{n}$ for $\chi^{m}$, that

$$
\left\|u_{t t}^{n}\right\|_{L^{\infty}\left(H_{p}^{\prime}\right)} \leqslant C \text {. }
$$

Thus, $\left\{u^{n}\right\},\left\{u_{t}^{n}\right\}$, and $\left\{u_{t t}^{n}\right\}$ are uniformly bounded sets in $L^{\infty}\left(H_{p}^{1}\right)$. In particular $\left\{u^{n}\right\}$ is uniformly bounded in $H^{1}(I \times J)$, which is compactly contained in $L^{2}(I \times J)$. There thus exist $u \in L^{\infty}\left(H_{p}^{1}\right)$ and a subsequence $\left\{u^{n_{s}}\right\}_{\nu=1}^{\infty}$ such that as $\nu \rightarrow \infty, u^{n_{3}} \rightarrow u$ weakly* in $L^{\infty}\left(H_{p}^{1}\right)$, strongly in $L^{2}(I \times J)$, and almost everywhere, and such that $u_{t}^{n_{3}} \rightarrow u_{t}$ and $u_{t t}^{n_{3}} \rightarrow u_{t t}$ weakly* in $L^{\infty}\left(H_{p}^{1}\right)$. It follows that $u \in$ $C^{1}\left(H_{p}^{1}\right)$, and it is then easy to pass to the limit in the $L^{1}(J)$-weak* topology in (A.1), verifying (1.3) with $\chi=\chi^{m}$. Since $m$ is arbitrary, this completes the proof.

Proof of Theorem 1.2. We shall show that the sequence $\left\{u^{n}\right\}$ constructed above satisfies the inequality

$$
\left\|u^{n}\right\|_{L^{\infty}\left(H_{p}^{k}\right)}+\left\|u_{t}^{n}\right\|_{L^{\infty}\left(H_{p}^{k}\right)} \leqslant C
$$

with $C$ independent of $n$. Since the set of functions in $L^{\infty}\left(H_{p}^{k}\right)$ with norm not exceeding $C$ is compact, the result (1.4) follows. For $k=1$, the inequality (A.4) is given by (A.2). We shall proceed by induction on $k$, abbreviating $\partial^{k} / \partial x^{k}$ to $\partial^{k}$ and dropping the superscript $n$ for brevity. Replace $\chi^{m}$ in (A.1) by $\chi=(-1)^{k} \partial^{2 k} u_{t}$. 
Then, in view of the inductive hypothesis,

$$
\begin{aligned}
A\left(u_{t}, \chi\right) & =A\left(\partial^{k} u_{t}, \partial^{k} u_{t}\right)+\sum_{j=1}^{k}\left(\begin{array}{l}
k \\
j
\end{array}\right)\left[\left(\partial^{j} a \cdot \partial^{k+1-j} u_{t}, \partial^{k+1} u_{t}\right)+\left(\partial^{j} c \cdot \partial^{k-j} u_{t}, \partial^{k} u_{t}\right)\right] \\
& \geqslant \frac{1}{2 K}\left\|\partial^{k} u_{t}\right\|_{1}^{2}-C\left(\left\|u_{0}\right\|_{k}\right),
\end{aligned}
$$

where $K=\min (\inf a$, inf $c)$. Also,

$$
\begin{aligned}
\left|\left(\alpha(u) u_{x}, \chi_{x}\right)\right| & =\left|\sum_{j=0}^{k}\left(\begin{array}{l}
k \\
j
\end{array}\right)\left(\partial^{j}[\alpha(u)] \partial^{k+1-j} u, \partial^{k+1} u_{t}\right)\right| \\
& \leqslant \frac{1}{8 K}\left\|\partial^{k+1} u_{t}\right\|^{2}+C\left(\left\|u_{0}\right\|_{k}\right)\left(1+\|u\|_{k+1}^{2}\right) .
\end{aligned}
$$

Similarly,

$$
\left|\left(\beta(u) u_{x}, \chi\right)\right|+|(\gamma(u), \chi)| \leqslant \frac{1}{8 K}\left\|\partial^{k+1} u_{t}\right\|^{2}+C\left(\left\|u_{0}\right\|_{k}\right)\left(1+\|u\|_{k+1}^{2}\right) .
$$

Thus,

$$
\left\|u_{t}\right\|_{k+1}^{2} \leqslant C\left(\left\|u_{0}\right\|_{k}\right)\left(1+\|u\|_{k+1}^{2}\right) \text {. }
$$

It follows from Gronwall's lemma that

$$
\|u\|_{L^{\infty}\left(H_{p}^{k+1}\right)} \leqslant C\left(\left\|u_{0}\right\|_{k+1}\right) \text {. }
$$

These two inequalities imply (A.4) with $k$ replaced by $k+1$ and complete the proof.

Department of Mathematics and Institute for Physical Science and Technology

University of Maryland

College Park, Maryland 20742

Department of Mathematics

University of Chicago

Chicago, Illinois 60637

Department of Mathematics

Chalmers University of Technology and the University of Göteborg

Göteborg, Sweden

1. T. B. Benjamin, J. L. Bona \& J. J. Mahony, "Model equations for long waves in nonlinear dispersive systems," Philos. Trans. Roy. Soc. London Ser. A, v. 272, 1972, pp. 47-78.

2. J. L. Bona, W. G. Pritchard \& L. R. Scotr, "A comparison of laboratory experiments with a model equation for water waves." (To appear.)

3. J. L. BonA \& R. SmITH, "The initial-value problem for the Korteweg-deVries equation," Philos. Trans. Roy. Soc. London Ser. A, v. 278, 1975, pp. 555-601.

4. J. C. EILbeCK \& G. R. MCGurRe, "Numerical studies of the regularized long-wave equations I: numerical methods," J. Computational Phys., v. 19, 1975, pp. 43-57.

5. R. E. EWING, "Numerical solution of Sobolev partial differential equations," SIAM J. Numer. Anal., v. 12, 1975, pp. 345-363.

6. R. E. EwING, "Time-stepping Galerkin methods for nonlinear Sobolev partial differential equations," SIAM J. Numer. Anal., v. 15, 1978, pp. 1125-1150.

7. W. H. FoRD, "Galerkin approximations to non-linear pseudoparabolic partial differential equations," Aequationes Math., v. 14, 1976, pp. 271-291.

8. W. H. FORD \& T. W. TING, "Stability and convergence of difference approximations to pseudo-parabolic partial differential equations," Math. Comp., v. 27, 1973, pp. 737-743.

9. W. H. FORD \& T. W. TING, "Uniform error estimates for difference approximations to nonlinear pseudo-parabolic partial differential equations," SIAM J. Numer. Anal., v. 11, 1974, pp. 155-169. 
10. H. GAJEWSKI \& K. ZACHARIAS, "Zur starken Konvergenz des Galerkinverfahrens bei einer Klasse pseudo-parabolischer partieller Differentialgleichungen," Math. Nachr., v. 47, 1970, pp. 365-376.

11. P. Henrici, Discrete Variable Methods in Ordinary Differential Equations, Wiley, New York, 1962.

12. J. L. Lions, Quelques Méthodes de Résolution des Problèmes aux Limites Non Linéaires, Dunod, Paris, 1969.

13. L. A. Medeiros \& M. Milla Miranda, "Weak solutions for a nonlinear dispersive equation," $J$. Math. Anal. Appl., v. 59, 1977, pp. 432-441.

14. L. A. Medeiros \& G. Perla Menzala, "Existence and uniqueness for periodic solutions of the Benjamin-Bona-Mahony equation,” SIAM J. Math. Anal., v. 8, 1977, pp. 792-799.

15. M. Milla Miranda, "Weak solutions of a modified KdV equation," Bol. Soc. Brasil. Mat., v. 6, 1975, pp. 57-63.

16. D. H. Peregrine, "Calculations of the development of an undular bore," J. Fluid Mech., v. 25, 1966, pp. 321-330.

17. M. A. RAuPP, "Galerkin methods applied to the Benjamin-Bona-Mahony equation," Bol. Soc. Brasil. Mat., v. 6, 1975, pp. 65-77.

18. R. E. Showalter, "Sobolev equations for nonlinear dispersive systems," Applicable Anal., v. 7, 1978, pp. 297-308.

19. R. E. Showalter \& T. W. TING, "Pseudo-parabolic partial differential equations," SIAM J. Math. Anal., v. 1, 1970, pp. 1-26.

20. L. WAHLBIN, "Error estimates for a Galerkin method for a class of model equations for long waves," Numer. Math., v. 23, 1975, pp. 289-303. 